\title{
The Application of the distal fistula Flushing on the treatment for Neonates with Necrotizing Enterocolitis
}

\author{
Ji-xue Zhao ${ }^{a}$, Xue-song Zhaob, Chun-yu Dong* \\ Department of Pediatric Surgery, the First Hospital of Jilin University, Changchun 130021, China \\ *Corresponding author e-mail: chunyudong0431@126.com,

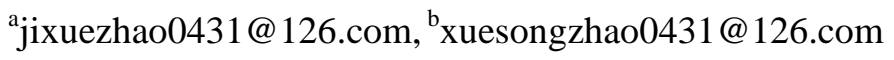

Keywords: Distal fistula flushing, Neonates, Necrotizing enterocolitis, Ileostomy.

\begin{abstract}
Object To explore the effects of distal fistula flushing on the treatment for the neonates with necrotizing enterocolitis. Methods: A retrospective analysis was conduct on 32 cases of newborns with necrotizing enterocolitis which need the ileostomy. All the patients were treated by single ileostomy or by ileostomy and distal fistula flushing between January, 2013 to January 2018. Duration of operation, amount of bleeding, the average length of stay and colonic stricture rate after operation were compared between the two groups, which were divided by treatment methods. Results: No significant difference was observed in age, and gender, duration of operation and amount of bleeding $(\mathrm{P}>0.05)$ between the two groups. However, the average length of stay was significantly shorter, the colonic stricture rate was significantly lower in the patients which were treated by ileostomy and distal fistula flushing $(\mathrm{P}<0.05)$. Conclusion: A retrospective analysis conducted by us showed the distal fistula flushing can significantly shorten the length of stay and reduce the colonic stricture rate for the patients with necrotizing enterocolitis which need the ileostomy.
\end{abstract}

\section{Introduction}

Neonatal necrotizing enterocolitis (NEC) is a common and serious intestinal disease in the neonatal period, especially in premature infants [1]. The disease is more commonly seen in premature infants, especially those with extremely low or ultra-low birth weight. NEC developes rapidly, and has high fatality rate and more complications, and it is still an important cause of death in the intensive care unit for neonates. The main pathological characteristics of NEC is the extensive hemorrhage necrosis of small intestine and the colon. Many NEC cases which have no intestinal necrosis and perforation can get conservative treatment during early stage, and many of them could get successful treatment which has the cure rate of $82.1 \%[2]$, but the data show that $20 \%-60 \%$ of the children still need the surgery[3]. The main operation method for the NEC cases is to perform the ileostomy, and 3 months later, ostomy closure will be performed.

While many cases with ileostomy still recovered slowly and many of them formed serious intestinal stricture. In view of this kind of situation, we adopted distal fistula flushing for the cases with ileostomy from January of 2013, and achived ideal clinical results.

\section{Patients and Methods}

\subsection{Patients}

When receiving the paper, we assume that the corresponding authors grant us the copyright to use the paper for the book or journal in question. Should authors use tables or figures from other Publications, they must ask the corresponding publishers to grant them the right to publish this material in their paper. From January of 2013 to January of 2018, 32 surgically treated neonates with necrotizing enterocolitis in the neonatology department, the First Hospital of Jilin University were 
enrolled in this retrospective analysis. Each patient signed an informed consent form. Approval was obtained from the institutional review committee of Jilin University.

\subsection{Study design}

The patients were divided into two groups according to the treatment methods: 15 patients were treated by traditional method, that is ileostomy; 17 patients were treated by ileostomy and distal fistula flushing, including the intraoperative flushing and postoperative flushing with saline. And all the cases were conducted colonography one month after operation.

\subsection{Methods}

Traditional treatment methods: Single ileostomy was applied in the patients with NEC, and then the conservative medical treatment was performed.

Improved treatment method: Compared with traditional treatment methods, we increased the distal fistula flushing, that is flushing the distal fistula with saline during the operation and flushing the distal fistula for 5-7 days after operation. And all the cases were conducted colonography one month after operation.

\subsection{Evaluation Criteria of Trentment Effects}

Evaluation criteria included the average length of stay and the colonic stricture rate after operation.

\subsection{Statistical Analysis}

All measured parameters including the average length of stay and the colonic stricture rate after operation, were analyzed by the statistical software program Statistical Product and Service Solutions (SPSS) 17.0 (SPSS Inc., Chicago, IL, USA) and expressed as mean \pm standard deviation $( \pm \mathrm{s})$, and t-test was used. Enumeration data including gender, prevalence frequency were analyzed by $\chi 2$ test. $\mathrm{P}<0.05$ was considered significant..

\section{Results}

\subsection{Baseline Characteristic}

There were no significant difference $(\mathrm{P}>0.05)$ in general data including operation age, gender between the two groups (Table 1.).

Table 1. Baseline characteristics of study patients

\begin{tabular}{cccc}
\hline Characteristic & control group $(15$ cases $)$ & experimental group $(17$ cases $)$ & $P$ value \\
\hline Operation age (days) & $8.8 \pm 3.4$ & $9.2 \pm 2.9$ & non-significant \\
Female & 5 & 6 & non-significant \\
Male & 10 & 11 & non-significant \\
\hline
\end{tabular}

\subsection{Evaluation of Treatment Effects}

All the cases of the two groups were survived. But the average length of experimental group was obviously shorter than that of control group $(\mathrm{P}<0.05)$. And all the cases were conducted colonography one month after operation. Among the cases of experimental group, 4 cases exist colonic stricture, and the colonic stricture rate is 23.5\%, Among the cases of control group, 11 cases exist colonic stricture, and the colonic stricture rate is $73.3 \%$. The colonic stricture rate of the experimental group was obviously lower than that of control group $(\mathrm{P}<0.05)$. Postoperative follow-up was conducted for 6 months. 
Table 2 Efficacy of the two groups

\begin{tabular}{cccc}
\hline & control group(15cases) & experimental group(17 cases) & $P$ value \\
\hline The average length of stay(days) & $22.5 \pm 5.4$ & $14.4 \pm 4.2$ & $P<0.05$ \\
colonic stricture rate & $11 / 15$ & $4 / 17$ & $P<0.05$ \\
\hline
\end{tabular}

\section{Discussion}

Necrotizing enterocolitis(NEC) is a common intestinal disease in the neonatal period, with intestinal inflammatory and necrotic changes as the main manifestations, and is a devastating intestinal condition with unclear causes, and a common acute abdominal disease in NICU[4]. NEC is a disorder characterized by ischemic necrosis of the intestinal mucosa, which is associated with inflammation, invasion of enteric gas forming organisms, and dissection of gas into the muscularis and portal venous system [5]. Early stage of NEC mostly could be cured by supportive treatment and antibiotics, but for serious cases which have existed intestinal necrosis or perforation, surgery in necessary. Surgical treatment, consisting in laparotomy and resection of the gangrenous bowel and/or ileostomy, is usually indicated in Bell stage III NEC. Peritoneal drainage has been advocated as a less invasive procedure in unstable VLBW patients[6], but laparotomy has recently revealed the treatment of choice[7].Among all of these surgical methods, resection of the gangrenous bowel and ileostomy are very common, many of the NEC cases could recovered gradually and wait for the next surgery--intestinal closure.

While, after ileostomy, many NEC cases still recovered unideally, including long-term parenteral nutrition, unmanageable infection and long-dated intestinal stricture. All of those are mainly because of the persistent existence of the residual toxins of the gut. As we now know, the cause and pathogenesis of NEC are not yet clear, it has been generally agreed in recent studies that the incidence of NEC is correlated with premature delivery, improper feeding, immature intestinal immune regulation, intestinal ischemia and hypoxia, abnormal bacterial colonization, and genetic factors [8]. The inflammatory cascade is caused by a variety of pathogenic factors, and the final common pathway is the damage to intestinal tissues resulting from inflammatory mediators [9]. So reducing the absorb of the toxins will controbute to the recover of NEC cases after operation. Aim at this situation, we adopted distal fistula flushing for the cases with ileostomy. We flush the distal fistula with saline during the operation and flush the distal fistula for 5-7 days after operation. And this type of surgical method achieved satisfactory clinical effects with a significant shorter average length of stay and lower colonic stricture rate of $23.5 \%$ compared to $73.3 \%$ of the control group.

Possible reasons were analyzed and listed as follows. On the one hand, since the main reason of the NEC is the toxin absorption and inflammatory reaction of the intestinal mucosa, we reduce the toxin absorption by flushing the distal fistula with saline during and after the operation, which will contribute to the recovery of NEC patients. On the other hand, strictures is a well-known common complication of NEC during posthealing process, affecting about $20 \%$ of NEC survivors [10]. After the acute episode of NEC, strictures develop at a variable period irrespective of the mode of management, which is associated with severe and prolonged morbidity (septicemia, perforation, intestinal obstruction) and morbidity secondary to intestinal strictures[11]. As we now know, the Post-NEC intestinal stricture is associated with the inflammatory reaction and damage of the intestinal mucosa, if the intestinal content persistently exist after ileostomy, and the toxin absorption and inflammatory reaction of the intestinal mucosa will exist for a long time, which will increase the risk of intestinal stricture. While the flushing of the distal fistula with saline during and after the operation could reduce the inflammatory reaction of the intestinal mucosa and promote the repair of intestinal injury, which will reduce the incidence of intestinal stricture. 


\section{Conclusion}

Distal fistula flushing can significantly shorten the length of stay and reduce the colonic stricture rate for the patients with necrotizing enterocolitis which need the ileostomy. And as an easy and feasible method for the NEC patients improved by us, has a satisfactory clinical effect, and it should be recommended.

\section{References}

[1] Eaton S, Rees CM, Hall NJ: Current research on the epidemiology, pathogenesis, and management of necrotizing enterocolitis. Neonatology, 2017;111: 423-30.

[2] Chorna IS, Razumnyi PK, Buryi OM, et a1. Non-invasive methods of control of the intestinal motility in surgical practice [J]. Klin Khir.1 999; (2):22-25.

[3] Thompson AM, Bizzarro MJ.Necrotizing enterocolitis in newborns: pathogenesis, prevention and management[J]. Drugs. 2008;68(9):1227-38.

[4] Hongri Zha, Binghong Zhang, Lei Shi. Influence of intestinal trefoil factor on Interleukin -1 in rats with colonitis [J]. Journal of Applied Clinical Pediatrics, 2009,24(7):513-514.

[5] Neu J. Necrotizing enterocolitis: the search for a unifying pathogenic theory leading to prevention. Pediatr Clin North Am 1996; 43:409.

[6] Ein SH, Marshall DG, Girvan D. Peritoneal drainage under local anesthesia for perforations from necrotizing enterocolitis. J Pediatr Surg 1977; 12:963-7.

[7] Rees CM, Eaton S, Kiely EM, Wade AM, McHugh K, Pierro A. Peritoneal drainage or laparotomy for neonatal bowel perforation? A randomized controlled trial. Ann Surg 2008; 248:44-51.

[8] Diego F. Nino, Chhinder P. Sodhi, David J. Hackam1,2Necrotizing enterocolitis: new insights into pathogenesis and mechanisms.Nat Rev Gastroenterol Hepatol. 2016,13(10): 590-600.

[9] HY Wu, Y Yang. A analysis on risk factors in necrotizing enterocolitis in newborns. Chinese And Foreign Medical Research.2013,11(32):38-39.

[10] Schwartz MZ, Hayden CK, Richardson CJ, et al. A prospective evaluation of intestinal stenosis following necrotizing enterocolitis. J Pediatr Surg 1982;17:764-70.

[11] Blakely ML, Lally KP, McDonald S, et al. NEC Subcommittee of the NICHD Neonatal Research Network. Postoperative outcomes of extremely low birth-weight infants with necrotizing enterocolitis or isolated intestinal perforation: a prospective cohort study by the NICHD Neonatal Research Network. Ann Surg 2005;241:984-9. 